\title{
La influencia del capital intelectual en la gestión educativa Juan Fanning García
}

\section{The influence of intellectual capital in educational management Juan Fanning Garcia}

Melba Rita Vásquez Tomás

Judith Soledad Yangali Vicente

Universidad César Vallejo, Perú

Autor por Correspondencia: rvasquez_25@hotmail.com,judithsyv@gmail.com

Fecha de recepción: 14 de Abril de 2017 - Fecha de aceptación: 30 de Junio de 2017

Resumen: La presente investigación estudio en qué medida un programa de fortalecimiento al capital intelectual mejora la gestión educativa de los docentes de la institución Liceo Naval Juan Fanning García en sus dimensiones gestión institucional, pedagógica, comunitaria y administrativa. Es importante indicar que se ha contemplado un plan de actividades diseñado en sesiones y talleres de mejora dirigidos a los docentes, quienes han demostrado dificultades en su práctica pedagógica, esto ha sido evidenciado en los resultados que arrojaron las encuestas aplicadas a los diversos actores educativos como parte de la etapa de autoevaluación del proceso de acreditación que la institución inició en el año 2014. La investigación es de tipo aplicada con un diseño experimental de nivel cuasi-experimental y enfoque cuantitativo. En el método de análisis de datos se utilizó la prueba de la U Mann Whitney. La población es de 85 docentes de los tres niveles y se aplicó en una muestra de 80 docentes. Llegando a las siguientes conclusiones: la aplicación de un programa de fortalecimiento al capital intelectual mejora la gestión educativa en la institución educativa.

Palabras Claves: gestión pedagógica; gestión institucional; gestión comunitaria; gestión administrativa; gestión educativa y capital intelectual

Abstract: The present study investigates the extent to which a program to strengthen intellectual capital improves the educational management of the teachers of the Liceo naval Juan Fanning García in its institutional, pedagogical, community and administrative management dimensions. It is important to indicate that a Plan of activities designed in sessions and improvement workshops aimed at teachers, who have shown difficulties in their pedagogical practice, this has been evidenced in the results of the surveys applied to the various educational actors as part of the selfevaluation stage of the process of accreditation that the institution began in 2014. The research is of applied type with an experimental design of quasi-experimental level and quantitative approach. In the data analysis method, the U Mann Whitney test was used. The population is 85 teachers of the three levels and was applied in a sample of 80 teachers. Coming to the following conclusions: the application of a program to strengthen intellectual capital improves educational management in the educational institution.

Key words: pedagogical management; institutional management; community management; administrative management; educational management and intellectual capital 


\section{Introducción}

La gestión educativa puede definirse como el conjunto de actividades y diligencias estratégicas guiadas por procedimientos y técnicas adecuadas para facilitar que las instituciones educativas logren sus metas, objetivos y fines educacionales.

La investigación tuvo como objetivo determinar la influencia del capital intelectual en la mejora de la gestión educativa de la institución Juan Fanning García para lo cual se partió de un diagnóstico situacional en cuanto a los logros en la gestión desde el año 2010 a la fecha, al notarse que los objetivos y metas propuestas en los documentos de gestión no han sido alcanzados a pesar de los diversos planes de mejora, proyectos y acciones ejecutadas en la institución.

En los resultados arrojados en las encuestas de autoevaluación de la gestión aplicadas a los diversos actores de la comunidad educativa permitieron evidenciar limitaciones en las dimensiones gestión pedagógica, institucional, comunitaria y administrativa por parte del capital intelectual de la institución.

Por lo que fue necesario que el investigador tomara la decisión de ejecutarse el programa "Fortaleciendo el capital intelectual" en donde se contempla un plan de acciones como:

a) La aplicación de una lista de cotejo para evaluar la gestión educativa en sus dimensiones.

b) Elaboración de planes de mejora orientada al soporte al desempeño docente, trabajo con las familias y la comunidad, uso de la información y recursos para el aprendizaje.

c) Ejecutar un programa de fortalecimiento al capital intelectual.

Todo esto con el propósito que el docente fortalezca sus capacidades y habilidades en el desarrollo de su quehacer educativo, evidenciándose en una mejora en la calidad de la gestión educativa.

La presente investigación busca dar solución a este problema con la aplicación de un Programa de Fortalecimiento dirigido al personal docente para mejorar la gestión educativa en sus dimensiones pedagógica, institucional, administrativa y comunitaria. Por otro lado fomentar la participación en la toma de decisiones por parte del capital intelectual.

La investigación es pertinente desde la perspectiva que aporta instrumentos y métodos de recolección de datos, así como la aplicación de un programa de "Fortalecimiento al capital intelectual", encaminadas a superar las limitaciones y dificultades del personal docente potenciando sus capacidades. La cual se evidenciará en el logro de las metas propuestas y en la satisfacción de la comunidad por el servicio educativo brindado por parte de la institución.

La investigación se fundamenta en el paradigma de la gestión educativa estratégica la cual se sustenta en tres ejes: (a) el pensamiento sistémico, donde se gestiona, organiza y se toma decisiones a nivel institucional, esto conllevará a establecer los objetivos, metas y visión de la escuela. (b) el aprendizaje organizacional, contempla convertir a las instituciones en organizaciones inteligentes a partir de un aprendizaje colectivo del personal y trabajo en equipo. 
(c) el liderazgo pedagógico, donde el director y los docentes deben promover el cambio institucional a través de un liderazgo transformacional teniendo en cuenta la visión, misión y valores de la organización, todo ello con el propósito de alcanzar la mejora continua de la gestión educativa a partir de una adecuada toma de decisiones y esto se logrará potenciando las capacidades del capital intelectual con la ejecución del programa de fortalecimiento.

Para lograr los objetivos propuestos en la investigación se consideró las bases teóricas de la gestión educativa donde Cassasus (2000) lo define "como la capacidad para articular los recursos del que dispone la organización para lograr lo que se desea" (p.25). Esto significa que el capital intelectual debe tener la capacidad de relacionar sus conocimientos, capacidades, habilidades, estrategias, talento con la estructura del sistema, los recursos disponibles y los objetivos de la organización de tal manera que se genere una conexión directa que lleve a la organización a alcanzar los estándares de calidad.

Según refiere Chiavenato (2009). La perspectiva del capital intelectual considera conservar el conocimiento, las organizaciones deben ofrecer un trabajo estimulante que proporcione a las personas experiencias y conocimientos.

El conocimiento que aportan sus directivos constituye la riqueza más importante de las organizaciones. Las organizaciones necesitan estrategias claras del área de RRHH para atraer, conservar y motivar a su personal. Las organizaciones necesitan transformarse en organizaciones de aprendizaje para emplear adecuadamente el conocimiento.

Para tener éxito en la era de la información, las organizaciones deben adoptar la perspectiva del conocimiento e invertir en él.

\section{El programa fue estructurado de la siguiente manera:}

a) Planificación y ejecución de planes de mejora dirigida al capital intelectual.

b) capacitación a través de talleres de fortalecimiento en gestión en las dimensiones pedagógica, institucional, administrativa y comunitaria.

\section{La metodología del programa se caracterizó por ser:}

a) resolutivo, se promovió la reflexión y el análisis a través del estudio de casos.

b) vivencial, se contrastaron y compartieron experiencias.

c) practico, a partir de las experiencias vividas por los participantes permitió proponer acciones de mejora.

Los mecanismos de evaluación del programa aplicados a los test de entrada y salida; la primera para identificar las capacidades previas y la segunda para cotejar las capacidades fortalecidas, en la evaluación de proceso se realizaron mediante trabajo aplicativo después de cada sesión del taller.

\section{Método}


El tipo de investigación es aplicada porque tiene como finalidad la solución de problemas práctico. El diseño de investigación es experimental de tipo cuasi-experimental en el cual se manipularan deliberadamente la variable independiente capital intelectual para analizar las consecuencias en la variable dependiente gestión educativa.

\section{La población}

El objeto de estudio está constituido por 85 docentes de los niveles de inicial, primaria y secundaria. Siendo la muestra de 40 docentes para el grupo experimental, dicha representatividad se dio en base a la intención de selección del investigador y 40 docentes que constituye el grupo control.

Tabla 1 Distribución de la población

\begin{tabular}{|c|c|c|c|}
\hline \multirow[t]{2}{*}{ Nivel } & \multicolumn{2}{|c|}{ Docentes } & \multirow[t]{2}{*}{ Total } \\
\hline & $\mathrm{H}$ & M & \\
\hline Inicial & 02 & 08 & 10 \\
\hline Primaria & 09 & 25 & 34 \\
\hline Secundaria & 12 & 29 & 41 \\
\hline Total & 23 & 62 & 85 \\
\hline
\end{tabular}

\section{Métodos de análisis de datos}

La técnica utilizada para la obtención de la información es la observación a partir del instrumento lista de cotejo que contiene ítems sobre los indicadores y dimensiones de la variable dependiente gestión educativa que fue aplicada al personal docente de la institución Juan Fanning García del distrito de Chorrillos mediante un pre-test y pos-test. Es importante mencionar que el instrumento tiene un nivel de confiabilidad aceptable con un valor de 0.843343. Según la prueba de Kuder y Richardson (KR 20) en el programa de Excel.

Después de la recogida de datos se procesó la información mediante el sistema SPSS y los estadísticos de la media aritmética y la desviación estándar. La prueba estadística seleccionada para probar la hipótesis es la prueba de U Mann-Witney, esta es una prueba no paramétrica, aplicada a dos muestras independientes.

\section{Resultados}

\section{Tabla 2.Prueba de hipótesis}




\begin{tabular}{|c|c|c|c|c|c|c|c|c|}
\hline & Grupos & $\mathbf{N}$ & $\begin{array}{c}\text { Rango } \\
\text { promedio }\end{array}$ & $\begin{array}{c}\text { Suma de } \\
\text { rangos }\end{array}$ & $\begin{array}{c}\text { U de Mann } \\
\text { Whitney }\end{array}$ & $\begin{array}{c}\text { W de } \\
\text { Wilcoxon }\end{array}$ & $\mathbf{Z}$ & $\begin{array}{c}\text { Sig. } \\
\text { asintótica } \\
\text { (bilateral) }\end{array}$ \\
\hline \multirow{3}{*}{$\begin{array}{c}\text { Gestión } \\
\text { Educativa } \\
\text { Pre Test }\end{array}$} & G Control & 40 & 43,08 & 1723 & \multirow{3}{*}{697} & \multirow{3}{*}{1517} & \multirow{3}{*}{$-1,003$} & \multirow{3}{*}{0,316} \\
\hline & $\begin{array}{c}\text { G } \\
\text { Experimental }\end{array}$ & 40 & 37,93 & 1517 & & & & \\
\hline & Total & 80 & & & & & & \\
\hline \multirow{3}{*}{$\begin{array}{c}\text { Gestión } \\
\text { Educativa } \\
\text { Pos Test }\end{array}$} & G Control & 40 & 20,5 & 820 & \multirow{3}{*}{0} & \multirow{3}{*}{820} & \multirow{3}{*}{$-7,741$} & \multirow{3}{*}{0} \\
\hline & $\begin{array}{c}\text { G } \\
\text { Experimental }\end{array}$ & 40 & 60,5 & 2420 & & & & \\
\hline & Total & 80 & & & & & & \\
\hline
\end{tabular}

La tabla 2 muestra los resultados obtenidos luego de aplicar la U de Mann - Whitney, donde se aprecia que el valor de la significancia asintótica bilateral ( $p$ ) es " $p=0,000$ "es menor que el nivel de significancia $(\square=0,05)$ luego del pos - test; por tanto se rechaza la hipótesis nula y se acepta la alterna la cual refiere que si existe una mejora significativa en la gestión educativa luego de aplicar el programa Fortaleciendo el capital intelectual en el grupo experimental.

Esto significa que luego de aplicar las sesiones y las actividades previstas para el programa, hay una mejora considerable en las dimensiones de la variable gestión educativa que realizan los docentes, ya que al aplicar el post test evidenciaron tener más claros sus enfoques y haber mejorado su práctica pedagógica como efecto de sus participaciones en el programa

Tabla 3.Gestión educativa. Grupo experimental: Post test y pre test Grupo Experimental

\begin{tabular}{lllll}
\hline \multicolumn{2}{c}{ Entrada } & \multicolumn{3}{c}{ Salida } \\
\hline & $\mathrm{f}$ & $\%$ & $\mathrm{~F}$ & $\%$ \\
\hline Inadecuada & 37 & 92.5 & 0 & 0.0 \\
Poco adecua 3 & 7.5 & 5 & 2.5 \\
Adecuada & 0 & 0.0 & 35 & 87.5 \\
Total & 40 & 100 & 40 & 100 \\
\hline
\end{tabular}

Fuente: base de datos

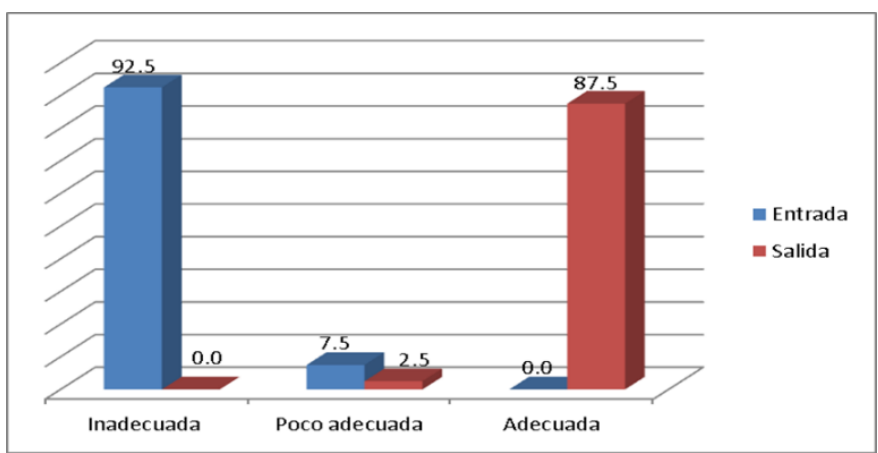

Figura 1. Gestión educativa. Grupo experimental: Post test y pre test - Fuente: base de datos

Tabla 4.Gestión educativa. Grupo control: Posttest y pre test 


\begin{tabular}{|c|c|c|c|c|}
\hline \multicolumn{5}{|c|}{ Grupo control } \\
\hline & \multicolumn{2}{|c|}{ Entrada } & \multicolumn{2}{|c|}{ Salida } \\
\hline & $\mathrm{f}$ & $\%$ & $\mathrm{f}$ & $\%$ \\
\hline Inadecuada & 33 & 82.5 & 26 & 65.0 \\
\hline Poco adecuada & 7 & 7.5 & 14 & 35.0 \\
\hline Adecuada & 0 & 0.0 & 0 & 0.0 \\
\hline Total & 40 & 100 & 40 & 100 \\
\hline
\end{tabular}

Fuente: base de datos

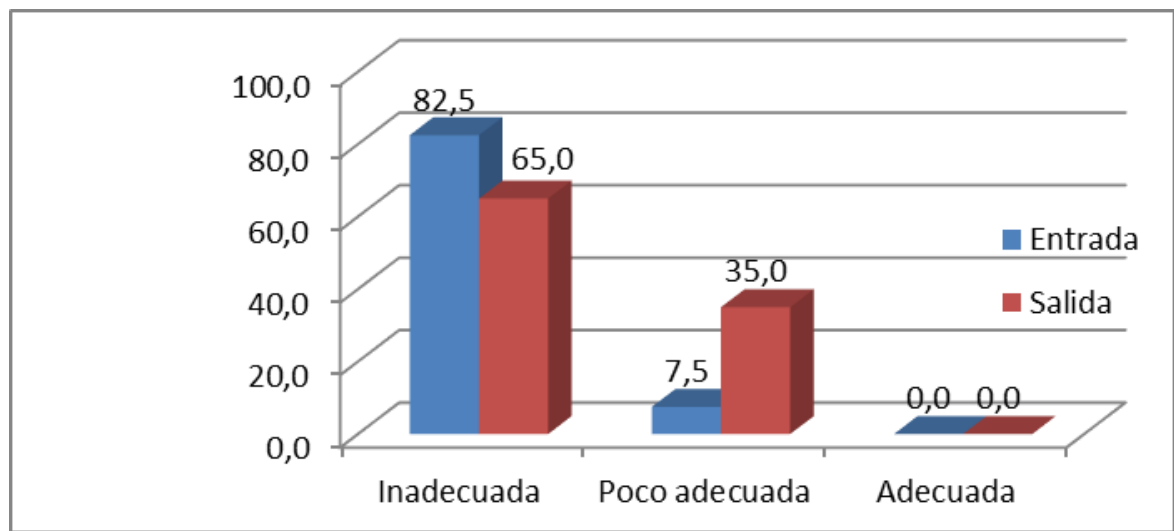

Figura 2.Gestión educativa. Grupo control: Post test y pre test

De acuerdo con la información recogida, la cual se organizó y presentó en la tabla 3 y figura 1, al comparar al grupo experimental en la prueba de entrada con la de salida, el resultado en la prueba de salida presentaba un mayor nivel si consideramos que un porcentaje significativo $87.5 \%$ tuvo un logro destacado y el $2.5 \%$ poco adecuado. Por otro lado, en la prueba de entrada los resultados indicaron que el $92.5 \%$ se encontraba en el nivel inadecuado, el $7.5 \%$ en el nivel poco adecuado. Mientras que la tabla 4 y figura 2 muestran que el grupo control en la prueba de salida alcanzó un resultado de $35 \%$ en el nivel poco adecuado y $65 \%$ de inadecuado a comparación de la prueba de entrada que arrojó un valor de $7.5 \%$ en el nivel poco adecuado y $82.5 \%$ en el nivel inadecuado

\section{Discusión}

La variable independiente presenta una significativa influencia sobre la variable dependiente debido a la ejecución de acciones y estrategias adecuadas. Sobre la influencia del capital intelectual podemos precisar que es significativa con respecto a las mejoras de la gestión educativa, en especial en las dimensiones institucionales, pedagógicas, comunitarias y administrativas evidenciadas en las pruebas aplicadas antes y después del programa experimental.

Después de aplicarse el programa en la I.E Juan Fanning García y el análisis de los resultados llegamos a las conclusiones siguientes: Con respecto al objetivo general se concluye que la influencia del programa "Fortaleciendo el capital intelectual es significativa en la mejora de la gestión educativa comparando los resultados del grupo experimental con relación al grupo control. En referencia a los objetivos específicos se concluye que la influencia del capital 
intelectual es significativa en la gestión pedagógica, seguida de la dimensión gestión institucional, la gestión comunitaria y administrativa se puede apreciar una mejora en la calidad del servicio brindado por la I.E Juan Fanning García.

Se recomienda aplicar la investigación y la propuesta del programa a otras instituciones educativas navales que forman parte de los colegios de la Marina de Guerra del Perú. Se Propone considerar dentro del PEI institucional proyectos de mejora de la calidad de la gestión orientado al capital intelectual. La institución debe incluir en sus proyectos institucionales a la comunidad y otras instituciones de la red 13 .

\section{Bibliografía}

Casassus, J. (2000). Problemas de la gestión educativa en América Latina. UNESCO.

Chiavenato, I. (2007). Administración de recursos humanos. El capital humano de las organizaciones. Mc Graw Hill Interamericana.

Edvinsson, L. y. (1999). El capital intelectual: como identificar y calcular el valor de los recursos intangibles de su empresa. España.

MINEDU/UNESCO (2011). Manual de gestión para directores de instituciones educativas. Gestión institucional. Lima.

Ley general de educación. (1999). Abedul. Lima.

Loera, S. (2004). Sistema de gestión de la calidad y capital intelectual en empresas estatales. España: Tesis de grado. Universidad Iberoamericana.

López, A. (2007). El trabajo en grupo de profesorado. España: Editorial Grao.

Morales, M. R. (2008). Gestión para la generación de capital intelectual en el contexto de la relación universidad-sector productivo. Venezuela: Tesis de grado. Universidad de Zulia.

OREALC/UNESCO. (2007). El derecho a una educación de calidad para todos en América Latina y el Caribe. Revista electrónica iberoamericana sobre calidad, eficacia y cambio en educación., 29-35. 